WE1.1 (Invited)

8:30 AM - 9:00 AM

\title{
Spatial and spectral nonlinear shaping of multimode waves
}

\author{
K.Krupa ${ }^{1,2, *}$, A. Tonello ${ }^{1}$, A. Bendahmane ${ }^{2}$, R. Dupiol' ${ }^{2}$, B. M. Shalaby ${ }^{1,3}$, M. Fabert ${ }^{1}$, A. Barthélémy ${ }^{1}$, G. Millot ${ }^{2}$, \\ S. Wabnitz ${ }^{4}$, and V. Couderc ${ }^{1}$ \\ ${ }^{1}$ Université de Limoges, XLIM, UMR CNRS 7252, 123 Avenue A. Thomas, 87060 Limoges, France \\ ${ }^{2}$ Université de Bourgogne Franche-Comté, ICB, UMR CNRS 6303, 9 Avenue A. Savary, 21078 Dijon, France \\ ${ }^{3}$ Physics Department, Faculty of Science, Tanta University, Tanta, Egypt \\ ${ }^{4}$ Dipartimento di Ingegneria dell'Informazione, Università di Brescia, and INO-CNR, via Branze 38, 25123 Brescia, Italy
}

*Email: krupa.katarzyna@yahoo.com

\begin{abstract}
We demonstrate a novel nonlinear dynamics of multimode fibers that reshapes their spectral and spatial beam profiles, based on spatiotemporal modulation instability. Sidebands ranging from the visible to the near-infrared are carried by one and the same spatial bell-shaped profile.
\end{abstract}

Keywords - nonlinear optics; multimode optical fibers; parametric processes; four-wave mixing; spatiotemporal dynamics;

\section{INTRODUCTION}

The spatiotemporal dynamics of beam propagation may enable the generation and manipulation of ultrabroadband light waves. Multimode fibers (MMFs) provide a natural platform for studying complex nonlinear optical interactions, offering novel opportunities to reshape coherent wave structures for numerous advanced applications. In this perspective, MMFs are currently receiving a renewed attention, as they may provide higher capacity for optical communications via space-division-multiplexing, and higher power for diffraction-limited laser sources [1]. Graded-index multimode fibers (GRIN MMFs) are of particular interest: only recently these fibers have permitted to experimentally observe multimode optical solitons, dispersive waves and supercontinuum generation when pumping with femtosecond light pulses in the anomalous dispersion regime [2-5].

Here we introduce a new regime for the spatiotemporal dynamics of multimode beam propagation, based on pumping with intense, quasi-CW laser beams a GRIN MMF operating in the normal dispersion regime. We demonstrate a new way to simultaneously control the frequency and modal content of multimode beams. Namely, we experimentally observed that the natural periodic self-imaging of multimode waves leads, via spatiotemporal modulation instability, to the generation of a series of spatially coherent sidebands with exceptionally large frequency shifts [6].

\section{RESULTS AND DISCUSSIONS}

In our experiments, we pumped a standard GRIN MMF by an amplified Nd:YAG microchip laser delivering 900ps pulses with the repetition rate of $30 \mathrm{kHz}$. Using a wide input beam of about $35 \mu \mathrm{m}$ in diameter, we excited a large number $(\sim 200)$ of guided modes. The upper red curve of Fig. 1 shows an example of output experimental spectrum generated by an input pulse with peak power $P_{P-P}=50 \mathrm{~kW}$. We observe a sequence of spectral peaks covering the whole range of visible wavelengths. These anti-Stokes sidebands have their counterparts on the Stokes side, which however in practice is heavily absorbed in silica fibers. Nonetheless, we were able to detect the first order Stokes peak. The spectral separation between the pump and the farthest peak exceeds a full octave. The generation of a sequence of bright sidebands, linking directly the near-IR to the visible domain, is permitted by the spatio-temporal modulation instability, which is quasi-phase matched in normal dispersion regime thanks to the periodic spatial breathing of the multimode beam along its propagation.

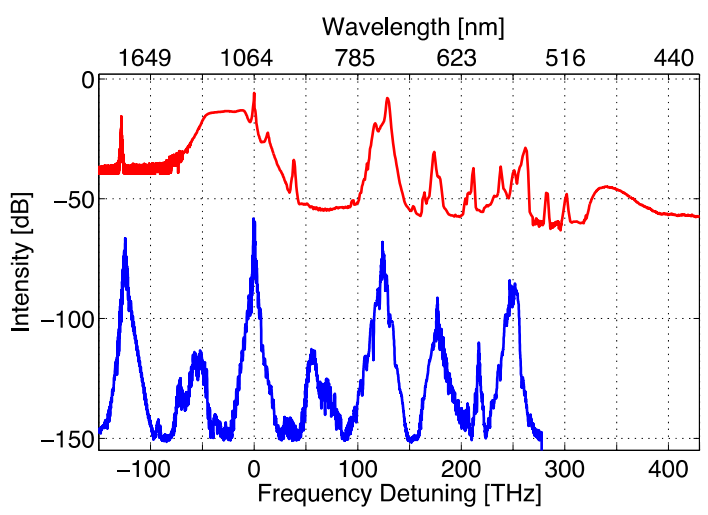

Fig.1. Experimental (upper, red curve) and numerical (bottom, blue curve) spectrum obtained in $6 \mathrm{~m}$-long GRIN MMF at $\mathrm{P}_{\mathrm{P}-\mathrm{P}}=50 \mathrm{~kW}$ and $0.22 \mathrm{~m}$-long GRIN MMF at $\mathrm{P}_{\mathrm{P}-\mathrm{P}}=125 \mathrm{~kW}$, respectively. Experimental spectrum (upper, red curve) includes also higher-order anti-Stokes peaks going out of the validity of the 3DNLSE model. Numerical spectrum has been vertically shifted for sake of readability. Simulations do not include Raman Scattering.

In fact, it is well known that a multimode beam experiences longitudinal oscillations when propagating through a parabolic GRIN MMF. The resulting periodic intensity variation leads, via the Kerr effect, to the generation of a self-induced periodic modulation of the refractive index: a series of multicolor waves can be then parametrically generated by the mechanism of quasi-phase matching. This natural self-imaging exhibits a sub-millimeter periodicity, which allows for the considerably large frequency shift of 123.5 THz for our first pair of sidebands. Whereas the frequency shifts of higher order sidebands grow larger in proportion with the square root of their index number. 
Our experimental results quantitatively agree very well with theoretical predictions [8], and with full numerical solutions of the space-time $(3+1) \mathrm{D}$ Nonlinear Schrödinger equation (3DNLSE), also known as the Gross-Pitaevskii equation in Bose-Einstein condensation: corresponding numerical results are shown in bottom blue curve of Fig.1.

We also investigated the spatial mode content of the output beam carried by each sideband: corresponding experimental results are illustrated in Fig.2. Strikingly, we observed that the entire spectrum accessible to our instruments exhibits a remarkably robust and spatially coherent nonlinear modal profile, in spite of the initially highly multimode excitation [7]. This unexpected Kerr-induced beam self-organization towards a quasi-Gaussian shape is fundamentally different from the spatial beam cleanup of the Stokes wave based on Raman or Brillouin scattering, which was previously reported in the literature $[9,10]$.
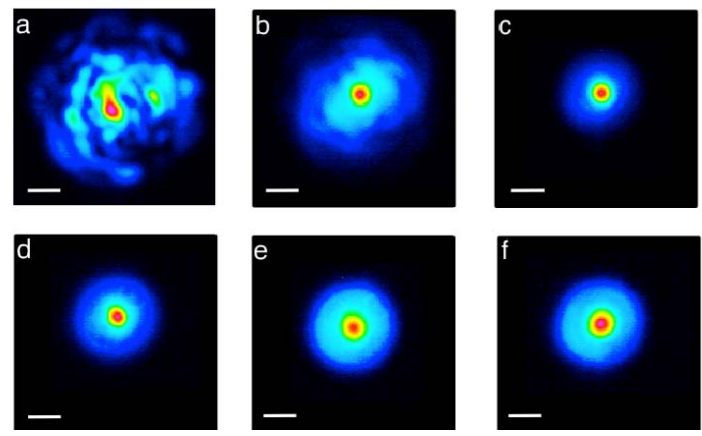

Fig.2. Experimental 2D output spatial profile at the pump wavelength of $1064 \mathrm{~nm}$ for an input powers: (a) $\mathrm{P}_{\mathrm{P}-\mathrm{P}}=0.06 \mathrm{~kW}$, (b) $\mathrm{P}_{\mathrm{P}-\mathrm{P}}=50 \mathrm{~kW}$, and at first four orders anti-Stokes sidebands at (c) $750 \mathrm{~nm}$, (d) $650 \mathrm{~nm}$, (e) $600 \mathrm{~nm}$, (f) $550 \mathrm{~nm}$ for $\mathrm{P}_{\mathrm{P}-\mathrm{P}}=50 \mathrm{~kW}$; Scale bar: $10 \mu \mathrm{m}$.

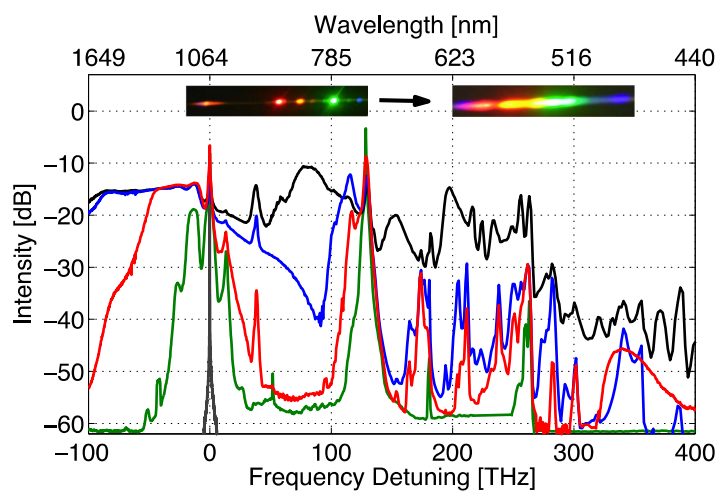

Fig.3. Experimental results of spectral evolution upon propagation distance $z$ at $\mathrm{P}_{\mathrm{P}-\mathrm{P}}=60 \mathrm{~kW}$ : (green curve) $\mathrm{z}=3 \mathrm{~m}$, (red curve) $\mathrm{z}=6 \mathrm{~m}$, (blue curve) $\mathrm{z}=12 \mathrm{~m}$; Black curve is measured at $P_{P-P}=50 k W$ and $z=12 \mathrm{~m}$; input pump spectrum is also reported for ease of comparison (grey curve).

In Fig. 3 we show that the spatiotemporal MI sidebands can, in turn, induce additional frequency sidebands by means of cascaded four-wave mixing. Such a complex interplay may further evolve into the generation of a wideband supercontinuum extending from the visible to the near-IR, still exhibiting a unique bell-shaped spatial modal profile. The photographic inset of Fig.3 shows an example of the transition from a discrete to a continuum spectrum. The competition between spatiotemporal MI and Raman scattering can be also observed in Fig.3.

\section{CONCLUSIONS}

We demonstrated a new method for directly converting a nearIR laser into an extremely wide range of optical frequencies, all carried by a spatially well-defined shape. The process is based on the Kerr effect, and it exploits the natural spatial selfimaging of multimode waves. Our results may enable new routes for a variety of technological innovations, including the original concept of energetic and ultra-broadband laser sources with a controllable spatial coherence of the beam for defense, spectroscopy and medical diagnostics applications. Our findings also considerably advance the emerging field of spatiotemporal multimode nonlinear dynamics, and may have significant consequences in other physical settings, where the Gross-Pitaevskii equation applies.

\section{ACKNOWLEDGMENT}

We acknowledge the financial support provided by Bpifrance OSEO (Industrial Strategic Innovation Programme) (Dat@diag project), Région Limousin (C409-SPARC), iXcore research foundation, Photcom Région Bourgogne, ANR Labex Action, and by the Italian Ministry of University and Research (MIUR) (2012BFNWZ2). We thank F. W. Wise, L. G. Wright, Z. Liu, B. Kibler, P. Tchofo-Dinda and A. Picozzi for valuable discussions.

\section{REFERENCES}

[1] D. J. Richardson, J. M. Fini, and L. E. Nelson, "Space division multiplexing in optical fibers", Nat. Photon. 7, 354-362, 2013

[2] L. G. Wright, D. N. Christodoulides, and F. W. Wise, "Controllable spatiotemporal nonlinear effects in multimode fibres," Nat. Photon. 9, 306-310, April 2015.

[3] L. G. Wright, S. Wabnitz, D. N. Chistodoulides, and F. W. Wise, "Ultrabroadband dispersive radiation by spatiotemporal oscillation of multimode waves", Phys. Rev. Lett. 115, 223902, 2015.

[4] W. H. Renninger and F.W. Wise "Optical solitons in graded-index multimode fibres," Nat. Comm. 4, article n¹719, 2013.

[5] A. Picozzi, G. Millot, and S. Wabnitz, "Nonlinear virtues of multimode fibre", Nat. Photon. 9, 289, 2015.

[6] K. Krupa, A. Tonello, A. Bendahmane, B. M. Shalaby, A. Barthélémy, V. Couderc, G. Millot, S. Wabnitz, "Observation of geometric parametric instability induced by the periodic spatial self-imaging of multimode waves", 2016 - accepted for publication in Phys. Rev. Lett.

[7] K. Krupa, A. Tonello, B. M. Shalaby, A. Barthélémy, G. Millot, S. Wabnitz, and V. Couderc, "Spatial beam self-cleaning in multimode fiber", http://arxiv.org/abs/1603.02972, 2016

[8] S. Longhi, "Modulational instability and space-time dynamics in nonlinear parabolic-index optical fibers", Opt. Lett. 28, 2363, 2003.

[9] N. B. Terry, T.G. Alley and T.H. Russell "An explanation of SRS beam cleanup in graded-index fibers and the absence of SRS beam cleanup in step-index fibers," Opt. Exp. 15, 17509, 2007.

[10] L. Lombard, A. Brignon, J.-P. Huignard and E. Lallier "Beam cleanup in a self-aligned gradient-index Brillouin cavity for high-power multimode fiber amplifiers," Opt. Lett. 31, 158, 2006. 